\title{
La enseñanza de la literatura en la modalidad de educación a distancia: una mirada desde sus actores en la Sede de Comayagua del Centro Universitario de Educación a Distancia de la Universidad Pedagógica Nacional Francisco Morazán
}

Maida Rosibel Ochoa Pineda*

\section{Resumen}

La modalidad de educación a distancia presupone las estrategias para la formación de estudiantes autónomos e independientes en sus aprendizajes; con la tarea del docente de planificar los cursos; seleccionar textos y materiales de estudio; escoger estrategias de aprendizaje y preparar las pruebas, entre otras. Por otro lado se resalta el auge que en las últimas décadas han alcanzado los sistemas a distancia, por las posibilidades que brindan a más adultos con compromisos familiares y de trabajo, de alcanzar un título universitario. Con la revisión de las actuales formas de enseñanza de la Literatura en la Sede del CUED en Comayagua en su tarea de la formación de docentes, se describe el rol del tutor; la participación del alumno; los recursos utilizados, las formas de mediación didáctica y las características de la sede, elementos de un sistema contemporáneo de educación a distancia.

Palabras Clave: <Educación a distancia o abierta $><$ Modalidad presencial $><$ Estudiante-Participante $><$ Estrategias de aprendizaje $>$ $<$ Mediación e Interacción Didáctica > <Profesor/tutor > <Formación de Formadores $><$ Lengua y Literatura $><$ Auto aprendizaje $><$ Estudio Independiente y Autónomo $><$ Innovación Tecnológica $><$ Paradigma Educativo > <Movilidad Estudiantil $>$.

*maidochoa@yahoo.com

<Paradigma> - Revista de Investigación Educativa. Año 20. No. 32 


\section{Origen y justificación de la investigación}

En el campo de la educación y por la ubicación del estudio se hace necesario revisar el rol que en la actualidad juega la educación superior o universitaria; y que en las palabras de Delors (2002, p. 162) [1], es la que mayor responsabilidad tiene en la formación de la experiencia, cultural y científica, acumulada por la humanidad y ha aumentado su importancia y la de las instituciones que se dedican a difundirla. De este modo, las universidades son las principales responsables de la idea del progreso, a través de la investigación, la innovación, la enseñanza y formación. En este marco, se encuentran muy difundidas las ventajas que ofrece la modalidad de educación a distancia porque permite a muchos jóvenes y adultos alcanzar un título y una formación que no habría sido posible años atrás, más si por diversas circunstancias no se tiene acceso a sistemas de educación presencial.

Debido a lo anterior el aporte teórico que conlleva el presente estudio, implica la evidencia empírica de la revisión de las actuales prácticas pedagógicas, a nivel metodológico y de formas de mediación que actualmente se están realizando, en la enseñanza de la Literatura en la Sede de la ciudad de Comayagua, bajo la responsabilidad de la Sección de Letras del Centro Universitario de Educación a Distancia -CUED- de la UPNFM y desde la mirada de sus actores: los docentes y estudiantes participantes en el estudio. Vale mencionar que la UPNFM es la única institución en el país encargada de la formación de docentes que luego prestarán sus servicios en los diferentes niveles educativos, desde preescolar hasta el nivel superior.

Con el estudio se pretende también contribuir a la sistematización de información que históricamente corrobore la experiencia de 34 años de enseñanza a distancia por parte del CUED en la propia experiencia de la investigadora que forma parte del cuerpo docente en la Sección de Letras y Lenguas, especialidad de Literatura.

Por otro lado, viviendo en carne propia cómo los docentes, pero sobre todo los estudiantes, presentan dificultades al adaptarse al sistema de distancia luego de toda una vida de presencialidad y debiendo enfrentar con madurez y autonomía un proceso de estudio 
en solitario (Barberá, 2001) [2]; la investigadora pudo corroborar cómo esta nueva forma de mediación didáctica muchas veces genera insatisfacción y hasta cierto grado de frustración. Sumado a lo anterior, las prácticas de los docentes de Educación a Distancia no siempre son adecuadas a las necesidades e intereses de los estudiantes, o a la novedad y circunstancia de generar formas de estudio independientes y autónomas, sin la presencia del docente.

\section{Objetivos de la investigación}

Constituyó el objetivo general de la presente investigación el conocer los mecanismos, formas y procedimientos en que se desarrolla el proceso de enseñanza de la Literatura en la Carrera de Letras y Lenguas en la Sede del CUED de Comayagua y la manera en que se comportan y relacionan los componentes, es decir, el profesor o tutor, el alumno o participante, la forma de mediación o interacción didáctica y la evaluación, los materiales o recursos y el soporte o sede en la modalidad de educación a distancia.

Los objetivos específicos que dirigieron la indagación son: Describir el rol del profesor o tutor de Literatura así como el desenvolvimiento del alumno o participante del programa; establecer la relación existente entre las formas de mediación o interacción didáctica con los materiales y recursos utilizados en la enseñanza de la Literatura en el proceso de Educación a Distancia; definir los roles de cada uno de los actores para asegurar el éxito o no de esta oferta educativa; describir desde la perspectiva de los actores en qué consiste el estudiar Literatura en una modalidad a distancia; establecer relaciones entre los componentes de la modalidad de educación a distancia, el profesor o tutor, el alumno o participante, la forma de mediación o interacción didáctica, la evaluación, los materiales o recursos y el soporte o sede en la enseñanza de la Literatura en el CUED de Comayagua; e, identificar los mecanismos, formas y procedimientos en que se desarrolla el proceso de enseñanza de la Literatura en la Carrera de Letras y Lenguas en la Sede del CUED de Comayagua. 


\section{Hacia una conceptualización de la educación a distancia}

El rasgo principal que caracteriza a las forma de Educación a Distancia es que ésta consiste en un tipo de educación en el cual los estudiantes y profesores están separados geográficamente (Noé 2003) [3]; así, "en el transcurso del proceso de enseñanza aprendizaje, al alumno se encuentra a cierta distancia del profesor" (Wedemeyer, 1973, en Holmberg, 1985, p. 11) [4]. Se llama "a distancia" por la separación física entre el docente y el alumno, o porque el componente mayoritario del proceso formativo se da a distancia; es "semipresencial" cuando el componente de presencia y distancia, es similar (Romero y Rubio, 2004, p. 175, en la compilación de Mena) [5].

Noé (2003), apunta que el primer esfuerzo por definir la educación a distancia que se conoce es la de G. Dohmen (1967), director del Instituto Alemán de Educación a Distancia (DIFF) en la antigua República Federal Alemana quien consideraba que la educación a distancia era una forma sistemáticamente organizada de auto estudio, en la que tutorías, la presentación de materiales de aprendizaje, y la supervisión y confirmación del éxito de los estudiantes se lleva a cabo por un grupo de profesores, cada uno de los cuales tiene responsabilidades. Noé (2003) fundamentado en los aportes de otros autores, (Raymond, 2000: Rumble, 1986), afirma además que el término educación a distancia (ED) apareció por primera vez en el catálogo de la Universidad de Wisconsin en el año 1982. Esta misma fuente revela que fue a través de Otto Peters y en el año 1968 que el término se popularizó, y comenzó a ser ampliamente utilizado en los Estados Unidos de América en la década de los 1980

Holmberg en 1977 aportó una definición más completa de educación a distancia. Para este autor el término implicaba varias formas de estudio a diferentes niveles que no están bajo la continua supervisión de tutores presentes con sus estudiantes en los salones de clases pero que de la misma forma se benefician de la planificación, orientación y dirección de una tutoría organizada.

En los últimos tiempos se ha definido la educación a distancia como 
un diálogo didáctico mediado entre el profesor y el estudiante que, ubicado en espacio diferente al de aquél, aprende en forma independiente (García, 2007) [6]. Según éste, siempre que haya separación física entre los alumnos y el profesor, que se utilicen otras formas de mediación educativa y haya una institución responsable de dar seguimiento al proceso, se estará haciendo educación a distancia. Estas otras formas de mediación son aquellas diseñadas para que el estudiante desarrolle una forma autónoma e independiente de autogestión, en donde él mismo es responsable de sus aprendizajes y el tutor es un guía o acompañante en el proceso.

Por lo tanto, para los fines del presente estudio, Noé (2003, p. 9) propone que una definición más completa de educación a distancia puede entenderse como: "el proceso educativo que propicia experiencias de enseñanza-aprendizaje a través de metodologías creativas que permiten sincrónica y asincrónicamente, a través de medios y herramientas técnico-tecnológicas, unir profesores y alumnos y que guiados por un conjunto de materiales bien estructurados proveen condiciones para un auto aprendizaje, una auto formación y una auto evaluación con una mínima dirección por parte del profesor".

Con la inclusión de las tecnologías de la información y la comunicación TIC se conoció una nueva forma de concebir el proceso de formación no presencial. El correo electrónico, la Web, los hipertextos, los CDROM se incorporaron a los programas educativos a distancia (Arancibia, 2002) [7].

Pero la adaptación a esta forma diferente de estudiar no siempre es satisfactoria a los estudiantes y puede producir frustraciones, Según Sánchez (2007) [8], "En muchas instituciones, que tienen esta modalidad educativa, existen cursos introductorios para dar a conocer este sistema, pero el profesor tutor debe continuar con este proceso de adaptación en las asesorías, tanto individuales como grupales o utilizando otros medios..." (p. 3). 


\section{La educación superior a distancia en Honduras y el papel protagónico del CUED}

En el estudio de Pineda (2011) [9], se menciona que según la Constitución Política vigente, uno de los principales objetivos de la Universidad Nacional Autónoma de Honduras es el "fomentar y promover el desarrollo de la Educación Superior en beneficio de la sociedad hondureña" (Art. 3, Ley Orgánica de la UNAH). Se menciona en el Art. 20 que la docencia se puede desarrollar por medio de dos modalidades de relación didáctica: presencial y a distancia. En el Art. 22 se lee que la modalidad a distancia es la que se desarrolla por medio del estudio autónomo conducente a la obtención de los objetivos educacionales planteados, correspondiendo al docente el rol de orientador o tutor y utilizando los recursos metodológicos específicos de la modalidad.

La Universidad Pedagógica Nacional Francisco Morazán (UPNFM) pionera en la modalidad de educación a distancia a través del Centro Universitario de Educación Distancia, (CUED), ha desarrollado un papel protagónico con la apertura de cada vez más sedes a nivel nacional, ha dado respuesta a la demanda de formación de docentes a nivel superior, desde sus inicios como Programa de Acción Comunitaria (PAC) en la Escuela Superior del Profesorado Francisco Morazán (ESPFM) ahora como Universidad Pedagógica Nacional Francisco Morazán (UPNFM) cumpliendo con la normativa antes mencionada. Surge como una respuesta a la demanda de formación docente en educación superior, el 15 de julio de 1978, en la ciudad de Choluteca, al sur de Honduras, en la institución predecesora de esta universidad, la prestigiada Escuela Superior de Profesorado.

La función de este centro universitario es la formación de docentes destinados a ocupar las plazas de docentes en los niveles de preescolar, primario y medio, e incluso educación en superior. Así, en el ámbito nacional, como se menciona en el documento Rediseño de la Carrera de Letras (2007) ) [10], la reforma educativa denominada Currículum Nacional Básico implementada por el Ministerio de Educación en la última década en el año 2002, hace énfasis en la enseñanza de la lengua, que aquí es llamada Área de Comunicación. 
En el seno del CUED, la Carrera de Letras y Lenguas comenzó a funcionar desde la creación misma de lo que fue la Escuela Superior del Profesorado Francisco Morazán en 1957, con el nombre de Letras y Ciencias Sociales. Hasta 1970 el Plan de estudios se culminaba en tres años. A partir de 1971 la estructura curricular sufre un cambio y se extiende el período de tres a cuatro años. Se optaba al título de Profesor de Educación Media en Letras y Lenguas. Al efectuarse la conversión de la Escuela Superior del Profesorado en Universidad Pedagógica, en 1989, el Plan sufre una nueva transformación y se otorga el título de Profesor de Educación Media en Letras en el grado de Bachiller Universitario.

A partir de 1994 se ejecutó una nueva reforma en el Plan de estudios, que incluyó la introducción de dos orientaciones para el estudiante de Letras: Linguística y Literatura. Con la última reforma a los planes y programas de estudio que entró en vigencia a partir de 2008, el ahora llamado Profesorado en la Enseñanza del Español, centra su actividad en torno a la enseñanza-aprendizaje de la lengua y la literatura españolas, con el énfasis en la formación didáctico pedagógica, la vinculación social y la investigación.

En esta función de formar profesores de Literatura, las habilidades lingüísticas mencionadas por Cassany (1997) [11], son las que el usuario de una lengua debe dominar para poder comunicarse, en un enfoque comunicativo se llaman destrezas o capacidades comunicativas. Así, profesor del área de Lengua y Literatura es, según Mendoza (2003) [12], el especialista en el lenguaje, que aporta los conocimientos necesarios para el desarrollo de las competencias comunicativas en todos los niveles escolares. En el caso específico de la enseñanza de la Literatura, fin del presente estudio, la didáctica moderna plantea justamente una didáctica especial de la misma, que deberá dar pautas para el desarrollo de la competencia literaria en los estudiantes.

En el caso de los docentes de literatura del CUED, no sólo desarrollan los contenidos programáticos en relación con las competencias plasmadas en los planes y programas de las diferentes asignaturas o espacios pedagógicos, sino que, implementan una serie de estrategias encaminadas a la motivación y estudio de la teoría 
literaria que le ayude a comprender no sólo los fenómenos y las corrientes o épocas de la historia de la literatura sino que también a despertar la pasión por la lectura de aquellos autores y obras literarias más significativas de la historia. Esta formación formará parte del tesoro cultural que acumulará a lo largo de su vida y que le dará la dimensión de un ser cada vez más humano y sensible ante la propia naturaleza humana.

La oferta académica de la Carrera de Letras en el Plan de Estudios 1994, ofrecía el llamado tronco común de asignaturas generales y pedagógicas. Entrando a la especialidad de Literatura cursaban espacios pedagógicos que iban desde las literaturas universales, europea, norteamericana, española, hispanoamericana, centroamericana y hondureña; se incluía además crítica literaria, semiótica, sociología de la literatura, temas y problemas de la literatura; igualmente se desarrollaba un espacio para la investigación documental de la literatura hondureña y la Práctica Docente que se sigue realizando en los institutos de educación media de carácter público.

\section{Los elementos de la educación a distancia}

Es necesario entender a la Educación a Distancia no como una serie de actividades desconexas, sino dentro de una compleja organización sistémica. Por tanto, estos elementos que la conforman, hacen parte de un sistema que integra los siguientes componentes en relación con sus objetivos. De este modo, el presente estudio se basará en el conjunto orquestado de los siguientes elementos:

\section{1 El docente o tutor}

Es aquí donde entra en juego, dentro de la calidad que debe tener la Educación a Distancia, el papel del docente, que se denomina tutor, en la administración de los saberes. Como se lee en el documento de Blanco (2007, p. 12) [13] sobre la reflexión de la calidad de la educación: "La calidad de los docentes y el ambiente que generan en la sala de clase, excluidas las variables extraescolares, son los factores más importantes que explican los resultados de aprendizaje de 
los alumnos, lo cual significa que las políticas orientadas a mejorar la calidad de la educación sólo pueden ser viables si los esfuerzos se concentran en transformar, con los docentes, la cultura de la institución escolar. A su vez, sin el concurso del profesorado ninguna reforma de la educación tendrá éxito".

De este modo, en los procesos a distancia la comunicación puede ser indirecta pero es fundamental el papel del profesor o tutor, quien constituye un elemento importante para el funcionamiento de los sistemas a distancia como responsable de crear los ambientes favorables a los aprendizajes de sus estudiantes, y que son distintos de la educación presencial.

El docente de esta modalidad, según Romero y Rubio (en la comp. de Mena, p. 190) debe tener, como todo profesor universitario, un conocimiento profundo y actualizado de su área específica y claridad sobre los fines educativos universitarios, así como un dominio de principios, teorías y metodologías de la Educación a Distancia, ahora se agrega, en forma cada vez más necesaria, el manejo de las TIC.

\subsection{El alumno o participante}

Holmberg (1985, p. 21) habla de las condiciones y problemas generales de los estudiantes a distancia, hace mención también de sus actividades típicas. "Mi concepción acerca del estudio a distancia, que permite a los estudiantes comenzar, interrumpir, continuar y terminar los cursos cuando lo deseen, evidentemente es atractiva para el estudiante autónomo, mientras que los sistemas regulados y con un ritmo definido parecerían imponer mayor dependencia a los destinatarios". Con pocas excepciones, estos estudiantes son adultos y en consecuencia, trabajan y tienen familia a cargo, como ya se mencionó en el presente estudio. Sigue Holmberg (1985, p. 27) “El estudiante a distancia está en una situación en la que tiene más probabilidades de seleccionar individualmente a qué debe dedicarse que los estudiantes convencionales, para quienes es obligatoria la asistencia a 
clase". Y esto es así porque está provisto de material para la auto instrucción, con el que trabaja solo, con una instrucción individualizada.

5.3 La metodología y formas de mediación en las enseñanzas a distancia, las estrategias y el proceso de evaluación en Educación a Distancia

En la educación a distancia, primero que todo el aprendizaje es básicamente una actividad individual, aun si trabaja en grupos colaborativos; y en cuanto a la enseñanza, "el estudio a distancia es una forma de educación que se basa específicamente en el trabajo personal de estudiantes individuales con mayor o menor grado de independencia respecto de la guía directa de los instructores" (Holmberg, 1985, p. 26). Por supuesto, lo anterior no significa, contrario a lo que muchos puedan pensar, que estudiar a distancia es estudiar sin profesor; en cambio, en la medida en que el estudiante atiende las explicaciones del tutor en los encuentros presenciales y cumple con sus tareas y asignaciones, logra experiencias exitosas de aprendizaje. Mejor es todavía la comunicación permanente y sistemática del estudiante con sus tutores vía teléfono, correo electrónico, las redes sociales y la mensajería de la Plataforma virtual.

En la actualidad, los modelos educativos de Educación a Distancia se caracterizan por la utilización de otros medios y formas de mediación además de la cita presencial, la lectura del texto oficial, además de lecturas complementarias como las obras literarias y la asignación de tareas entre un encuentro y otro. Para ello es necesario hacer una revisión de los métodos y formas de mediación que se están utilizando en los actuales procesos de Educación a Distancia.

La modalidad de Educación a Distancia es un sistema pedagógico que permite soslayar las limitaciones de espacio y tiempo, potenciando el autoaprendizaje, la interacción y la flexibilidad; y permitiendo el acceso generalizado a la educación superior y a la formación continua. Según Mena 
(2004), las características de los nuevos modelos y la descripción dominante de la Educación a Distancia, están fuertemente influidas por el papel de las TICs y la transformación que han traído a esta modalidad, con su inevitable camino a la virtualización; por una compleja red de componentes del nuevo paradigma; por la importancia de la dimensión pedagógica y por las variadas configuraciones estructurales que deberán adoptar las instituciones que generan y desarrollan los nuevos modelos.

De esta manera, el modelo pedagógico es precisamente la integración de los elementos de la Educación a Distancia para llevar a cabo los objetivos educativos y que son el motivo de análisis de esta investigación: una metodología adecuada así como las estrategias de aprendizaje y un sistema de evaluación que garantice el aprendizaje; los materiales y recursos; los profesores o especialistas, con una deseable preparación o especialización en Educación a Distancia; los alumnos participantes del programa y la infraestructura física -aquí llamada institución soporte-, que permita y facilite el proceso.

La Educación a Distancia se define, por tanto, como una educación que implica la separación física y una forma de trabajo en que no necesariamente coinciden en tiempo y espacio los estudiantes y sus profesores, de manera que se favorece el aprendizaje autónomo. Así, la paradoja a distancia querrá más bien significar sin distancia obedeciendo a una forma de formación que permite a más estudiantes, mayores posibilidades de acercamiento, mayor equidad e igualdad.

En la concepción de Arancibia y Pérez, el antecedente inmediato de la EaD es la educación presencial. La evolución de la modalidad presencial a la de a distancia, pasa también por la generalización y difusión de los llamados mass media, principalmente la radio y la televisión así como los medios de comunicación tradicionales como el teléfono y el correo postal. 
Especial atención requiere, en los aprendizajes a distancia, la selección y el uso del material impreso, todavía inseparable del alumno a distancia; es más, considerado inclusive, indispensable. Según Viesca (1999) [14], los materiales educativos en la educación a distancia tienen una gran importancia, antes que nada porque son medios de comunicación, y además, porque la posibilidad de apoyar los ambientes de aprendizaje en esta modalidad depende, en gran parte, de las posibilidades y uso que se haga de ellos.

A pesar de las nuevas tecnologías, se sigue y se seguirá recurriendo a los libros impresos, aun las universidades virtuales se apoyan en ellos. Al terminar la carrera, el alumno de distancia dispone de una bibliografía básica que le permite defenderse en el mundo del trabajo, a la que recurre en caso de necesidad y ante la escasez de bibliotecas especializadas. Dichos textos pueden ser de dos tipos: el llamado "texto básico" o material fundamental, generalmente obligatorio, elaborado por la institución o adquirido en alguna editorial y las guías didácticas o académicas, que son diseñados expresamente para orientar el aprendizaje en cada asignatura.

Como explica Viesca (1999, p. 9) “El estudiante a distancia va a trabajar con sus materiales en su casa, en la oficina, en un café, en muy diversos espacios que probablemente poco tengan que ver con el ambiente "ideal" de aprendizaje". Por lo tanto, lo que desea deba alcanzar en los aprendizajes, las destrezas y procesos mentales que se busca desarrollar, el volumen de la información accesible, las fuentes de información, entre otros, deberán estar indicados y contenidos en los textos.

\subsection{La infraestructura: institución de soporte y de gestión}

Por su naturaleza y características, la educación a distancia se dirige a un tipo de estudiante disperso geográficamente 
por el ámbito nacional y en su mayoría limitados a asistir a clases presenciales. Para ello, la institución gestora de una modalidad de Educación a Distancia, deberá contar con una Sede central o matriz, (Romero y Rubio, 2004) con una Dirección General o similar y las diferentes facultades, escuelas, secciones o departamentos y sedes regionales, donde se llevan a cabo los encuentros y en fin, todos los procesos académicos.

Las grandes universidades a distancia, como señalan (Romero y Rubio, 2004, p. 198) marcaron la pauta de la creación inicial de los Centros Universitarios o Centros Asociados como es el caso de la UNED española, cuyos fines específicos, entre otros son los de servir de nexo académico y administrativo entre la Universidad y los estudiantes; para el alumno, tramitar su matrícula, entrega y retira sus trabajos a distancia y realiza las evaluaciones presenciales, actividad que es administrada celosamente desde la Sede Central; brindar servicio de información y asesoría a los estudiantes.

Finalmente y siempre en la idea de Romero y Rubio (2004), es necesario agregar que tanto la Sede Central como los centros regionales, sedes y subsedes, deben tener la infraestructura adecuada y pertinente para el cumplimiento de sus funciones, como ser aulas amplias, iluminadas, ventiladas y limpias; el mobiliario suficiente y adecuado; biblioteca, fotocopiadora, laboratorio de computación, un centro de audiovisuales o sala de usos múltiples; además de los baños, la cafetería y la librería/papelería. Loable será el acceso gratuito o a bajo costo a los servicios de Internet, claves actualmente para la comunicación y la mediación tecnológica.

\section{El modelo y el diseño de la investigación}

Por las características de la indagación y los datos obtenidos, el tipo de enfoque de investigación es cualitativo. Dicho enfoque ha conllevado un proceso inductivo mediante el ingreso al ambiente o campo de la investigación en una acción recurrente, en donde las 
etapas son acciones que han permitido adentrarse en el problema de investigación y la consiguiente recolección de los datos en forma permanente.

Los objetivos y las interrogantes aquí planteadas son enunciativos y constituyen el punto de partida de la investigación ya que se ha querido conocer y describir la situación actual de la enseñanza de la literatura en la modalidad de la educación a distancia en la Sede de Comayagua. De esta forma, el planteamiento es expansivo pues ha ido enfocando conceptos relevantes de acuerdo con los hallazgos del estudio, en torno a los aspectos anteriormente mencionados, fundamentados en la teoría y con base en la experiencia de la propia investigadora.

Se ha pretendido tomar en cuenta y aprender de las experiencias y puntos de vista de los docentes de literatura, así como la opinión de los estudiantes participantes del proceso, valorar procesos y generar conclusiones en la perspectiva de los participantes.

El tipo de investigación realizado corresponde a una investigación narrativa-descriptiva ya que se ha recolectado datos basados en las experiencias propias de los docentes y estudiantes. El producto final del estudio (Carpio, 2006) [15] lo constituyen los datos obtenidos a partir de descripciones literales completas del asunto a estudio y de las características contextuales del escenario donde se protagonizan las acciones.

Es inductivo porque las generaciones, conceptos, categorías y teorías, entre otras, que aquí se reportan, emergen del examen concienzudo de la información, vinculando ésta con el contexto mismo donde se produjo la recolección de los datos.

Las unidades de análisis a tomar en cuenta son los ya mencionados elementos participantes en un proceso de Educación a Distancia.

El lugar en donde se desarrolló la investigación es la Sede del CUED de Comayagua en donde se sirve la Carrera de Letras y Lenguas, en la sub-especialidad de Literatura, del Plan de estudios 1994. La Sede donde se realiza la actividad académica está ubicada en el local rentado del Liceo Jesús de Nazaret, Barrio Arriba, Avenida 1 
NE, zona muy céntrica de la ciudad de Comayagua, cabecera del departamento del mismo nombre, que a su vez se localiza en el Valle de Comayagua, en el corazón de Honduras, América Central.

La conveniencia, accesibilidad y viabilidad de las condiciones responden a los objetivos del estudio en cuanto a la definición del ambiente, los participantes, la actividad educativa que se realiza, las situaciones y vivencias en la enseñanza de la literatura, etc. por lo que fue factible y conveniente la realización de la recolección de los datos.

Esta etapa de la inmersión total implicó una serie de actividades destinadas a la observación de los eventos que ocurren en el ambiente; al establecimiento de vínculos con los participantes; a la adquisición del punto de vista interno con respecto a la propia experiencia docente en la enseñanza de la Literatura; a la recolección de los datos sobre conceptos, formas de expresión y naturalmente, todo tipo de materiales impresos.

\section{Los sujetos y participantes}

Los sujetos en este estudio lo constituyen los docentes de literatura asignados a servir los espacios pedagógicos del plan de estudio de la carrera de Letras y Lenguas y los estudiantes inscritos en dicha carrera. Se inició el proceso de investigación en el III Período académico 2008 y continuó en los subsiguientes períodos académicos del año 2009. El diseño de investigación previó además, el retorno al ambiente de investigación cuantas veces fuera necesario. Se han identificado como participantes en investigación a los 5 docentes de la orientación de Literatura, que muy amablemente completaron los cuestionarios y permitieron el acceso de la investigadora a sus aulas para la observación de sus propias clases; y un grupo de estudiantes voluntarios, que accedieron a la invitación de la investigadora a colaborar completando los cuestionarios con la información requerida y que también mostraron su interés a toda forma de conversación y observación de su participación en clase, por la investigadora. 


\section{La recolección de datos y análisis de la información}

Los instrumentos que se utilizaron para los fines de la recolección de los datos de la presente investigación, se describen así: Diario de anotaciones de campo, cuaderno de apuntes en donde se registra los eventos o sucesos vinculados al planteamiento; diagnóstico inicial, escrito, a manera de exploración, con que se obtuvo un panorama general de la viabilidad de la investigación así como de la situación actual de la forma en que se está enseñando la Literatura en la Sede de Comayagua; observaciones estructuradas del desarrollo de visitas o tutorías (Reyes, 2008) [16], para conocer, con más detalles, el desarrollo de la actividad docente, también para explorar los ambientes de aprendizaje desarrollados en este sistema de Educación a Distancia; entrevistas estructuradas: con pautas de preguntas abiertas, con cuestionario -Guión de la entrevista- administrado por la entrevistadora (Reyes, 2008), definida como intercambio de información entre el investigador y los participantes seleccionados.

Fue interesante recurrir a la interrogación porque así se conoció, por la propia boca de los involucrados, sus prácticas, experiencias, opiniones, creencias, ideas, sentimientos, motivaciones, etc. referente a su rol tanto como docente o como estudiante de educación a distancia. En vista de que en esta indagación cualitativa la mayor riqueza de datos obtuvo de la observación durante la inmersión en la propia Sede y de las entrevistas a los participantes; debido además a la variedad de estas fuentes de información, se hizo triangulación de datos, para concretar el análisis de la información.

La triangulación se realizó de acuerdo con la información proporcionada por los participantes, de acuerdo con las categorías de análisis en cuanto a coincidencias y contrariedades (Carpio, 2006) relacionadas con las opiniones de los docentes de literatura en cuanto a su desempeño docente; las opiniones de los estudiantes participantes en cuando a cómo reciben la enseñanza por parte de los docentes y; la verificación de la investigadora de los anteriores datos de acuerdo con los resultados del diario de campo, de las observaciones directas y de las entrevistas individuales y grupales. 


\section{Resultados del estudio}

Según Sandín (2006), algunos investigadores consideran que los datos no deben ser analizados de por sí sino que la información conseguida debe presentarse tal como ha sido expresada por los informantes, con el entendido de que dicha información proporciona la verdadera visión de la realidad. Desde esta perspectiva, la investigadora presenta aquí los datos tal como fueron expresados por los estudiantes y docentes participantes en el estudio, en consonancia con el concepto de educación a distancia y los elementos de esta modalidad considerados en la presente indagación.

Dentro de los hallazgos quizá sea primordial dar prioridad al parecer de los participantes en cuanto a lo que significa estudiar a distancia, a diferencia de la modalidad presencial y se expresaron de la siguiente manera: significa la oportunidad de prepararse; un reto; aun si estudiar a distancia es más sacrificado que presencial, el esfuerzo radica principalmente en el estudiante, quien tiene que investigar por su cuenta, en Internet y bibliografía que esté a su alcance. Es importante destacar la diferencia expresada por los estudiantes en cuanto a formas de estudio presencial y a distancia, en el sentido que son muchas las dificultades que presentan los inscritos en el CUED cuando deben enfrentar una experiencia de estudios "a distancia", luego de toda una vida de estudios en forma "presencial" por el hecho de que la mayor responsabilidad recae en ellos mismos, debiendo volverse autónomos y gestores de sus propios aprendizajes.

En cuanto a las actividades habituales que desarrollan dentro de la tutoría o visita, tanto en el desarrollo de la clase como para la evaluación, los estudiantes manifestaron que durante una visita se revisa los trabajos de investigación; se hace plenarias del trabajo de los grupos; reuniones grupales; revisión de avances; pruebas; discusiones de obras literarias; exposiciones; controles de lecturas; ensayos. También se asignan los trabajos para hacer en casa.

Mientras que las tareas y asignaciones que les dejan para hacer en casa, se mencionaron los trabajos prácticos, investigaciones, lecturas de textos; lecturas, ensayos y presentaciones; investigaciones por Internet, en enciclopedias, sobre obras 
literarias; sumado a escritos y ensayos. Como puede verse, entre las actividades desarrolladas dentro de la tutoría se da importancia al trabajo grupal tanto en la tutoría como fuera de ella; a la revisión y discusión de avances en las investigaciones; a las exposiciones, plenarias y discusiones; realizan además pruebas presenciales y controles de lecturas de las obras literarias asignadas para lectura. En cambio, para hacer en casa se mencionan los trabajos prácticos e investigaciones, para las que recurren al Internet y a los textos a su alcance.

Referente al tipo de materiales, textos, recursos tradicionales, recursos tecnológicos, que se utilizan en el desarrollo de las clases 0 tutorías, los estudiantes mencionaron, en primer lugar el uso del texto [oficial] de la universidad, además de otros textos [tanto de teoría literaria como de obras literarias], Internet, lecturas complementarias, libros de texto y obras literarias, enciclopedias y el Internet; se agrega el Data Show, los marcadores, pizarra y computadora.

En definitiva, los recursos utilizados se reducen a los textos oficiales adoptados o preparados en el CUED, además de lecturas complementarias, que en su mayoría son libros de teoría literaria puestos a disposición por los mismos docentes y que generalmente deben fotocopiarse; y por supuesto las obras literarias.

Pasando a las funciones que desempeña el docente o tutor de Educación a Distancia, en cuanto a lo manifestado por los estudiantes, el tutor explica, aclara dudas, orienta en los trabajos asignados; es facilitador, despeja dudas, explica en una forma más amena; reforzar cada una de las actividades que realizan, además de ya mencionada la función de aclarar dudas.

Evidentemente son varias las ventajas y las desventajas de los estudios a distancia, pero el compromiso personal que el estudiante adquiere por el tener que estudiar "en soledad" hace que éste asuma con mayor responsabilidad sus aprendizajes y maneje su tiempo en forma independiente y autónoma, aún con la desventaja de no tener siempre presente al docente o tutor en el acompañamiento docente. 
El siguiente aspecto analizado es la participación del estudiante de distancia, lo consideran sus deberes u obligaciones: cumplir con las visitas asignadas y realizar los trabajos que el profesor solicite; mucha responsabilidad y dedicación con mis clases; la constante lectura, una buena organización de estudio y disponibilidad de aprender son las mejores herramientas para poder sacar adelante a mis alumnos y a mi país; estudiar a diario, investigar y cumplir con todas las tareas asignadas, estar en contacto con el docente por cualquier duda; tener estudiados los contenidos, previo a la visita que se realizará, realizar las tareas asignadas, solicitar información, aclaraciones de dudas, sobre el contenido, asistir a todas las visitas programadas para el período académico; entre otras.

Es muy importante también, para el apoyo de la mediación didáctica, la comunicación efectiva entre los participantes de procesos a distancia. Entre una visita y otra, ésta se realiza por medio de teléfono fijo y celular, el correo electrónico y el fax. En torno a este aspecto, resulta interesante lo manifestado: en los últimos períodos tuve más comunicación por Internet, lo que facilitó mi trabajo al tener acceso a que el docente me aclarara dudas y me brindara sugerencias sobre el trabajo realizado, lo que me permitía corregir errores y mejorarlo. A la vez ahorré en gasto de impresión y empastados; el docente da el número de teléfono pero no lo contestan y algunos profesores no dan su número de teléfono ni sus correos electrónicos.

En esta forma "mixta" de hacer educación a distancia en el CUED, o sea, con actividades presenciales y otras a distancia, se hace necesario que tanto los docentes como los estudiantes pongan a disposición los medios con que cuentan para la mediación didáctica, como ha sido mencionado por los participantes, la telefonía fija y celular, el correo electrónico y el fax.

Otro elemento de que se vale la actividad académica a distancia es el uso de medios impresos, aquí llamados textos.

Para los fines de este estudio se inquirió en cuanto a cómo son los textos y la forma en que son utilizados. Práctica tradicional en el 
CUED es la elaboración de compilaciones, reproducidas luego con fotocopia; otros textos son adquiridos en alguna editorial e incluso muchos textos originales son reproducidos con fotocopia por no distribuirse en el país y porque la compra al exterior resultaría onerosa para el alumno. En el caso específico de la Sección de Letras, es una asignación de trabajo para los docentes, el agregado de guías didácticas y cronogramas de trabajo al inicio del texto, con el fin de orientar el aprendizaje. El estudiante, debiendo estudiar solo y muchas veces teniendo su residencia en zonas remotas del país, deberá organizar su tiempo para cumplir con sus obligaciones, contando con el material ofrecido en la librería de la universidad o fotocopiado en algún negocio cercano a la sede. Haciendo un resumen del tipo de texto utilizado, es interesante ver que los estudiantes se expresaron reiteradamente con respecto de la calidad de los mismos: copias borrosas y mal compaginadas; los textos de estudio que asigna la universidad a la asignatura son horrendos, deficientes, "churuncuyos", la mayor parte de ellos no son utilizados porque no son de calidad; no me motiva comprar textos fotocopiados, los pido prestados y le saco copia a los temas que me eran interesantes o los capítulos que el docente evaluaría; el maestro hace que utilicemos otros tipos de texto o fuente; los textos no son buenos, la letra es muy pequeña y borrosa, son mal compaginados y algunos docentes no saben ni cómo utilizarlos; la mayoría de los textos son fotocopias mal encuadernados, mal fotocopiadas (casi no se leen) y de diversos textos, lo cual no da uniformidad ni continuidad al texto, lo que genera confusiones graves, incluso en los docentes; generalmente son folletos mal editados, caros y en algunos casos no se utilizan; la mayoría de veces están mal impresos y redundantes; los textos son desfasados, antiguos y los mismos de todos los años. Dichos textos son utilizados para su lectura y análisis, en forma individual y en trabajos de grupos, asignando los capítulos a utilizar; los docentes se sirven de los mismos para administrar el conocimiento y de las mismas lecturas se realizan los exámenes y pruebas escritas.

Además de los elementos anteriormente mencionados propios de los procesos educativos a distancia, se encuentra el de la instituciónsoporte o institución gestora de la modalidad. A estas sedes llegan los estudiantes de todos los lugares. Entre las dificultades que 
encuentran los tutores en el desarrollo de su trabajo docente en esta Sede, se muestran las siguientes: carencia de un ambiente pedagógico que permita un aprendizaje efectivo; falta de materiales (ni siquiera marcadores para escribir en el pizarrón); falta de recursos didácticos y tecnológicos como libros, computadoras, Internet, Data show; edificios no adecuados para la enseñanza; clima caliente, sin climatización (aire acondicionado o ventiladores); frecuente interrupción de las clases por avisos y mensajes; alta contaminación por ruido, olores nauseabundos; falta de apoyo de los responsables de la sede al atender emergencias de cualquier tipo o necesidades de los estudiantes y por parte de las autoridades hacia maestros y alumnos; problemas de logística: retraso de viáticos, retraso de sueldos, sensación de inestabilidad laboral por parte del maestro por hora; no hay a disposición de los docentes una biblioteca especializada.

Otra fuente de información en la búsqueda de hallazgos en el presente estudio la constituyeron las observaciones directas a docentes durante el propio desarrollo de la tutoría, realizadas por la investigadora. Las transcripciones íntegras de las tutorías observadas, constituyen valiosos documentos para la documentación de la experiencia, así como la continuación de estudios relacionados. La observación directa no estructurada fue considerada en esta indagación, como una metodología cualitativa con el propósito de confrontar la experiencia docente de la propia investigadora, en su trayectoria como docente de literatura, primero como docente por contrato por hora, ahora de planta a tiempo completo, en el CUED. En la expectación se tomó en cuenta el ambiente del aula y las características del espacio en donde se desarrolla la clase o tutoría, la forma como es presentada e iniciada la misma; las actividades desarrolladas por el tutor; la participación, respuesta o comportamiento de los estudiantes ante las actividades propuestas por el docente; el tipo de materiales utilizados y las tareas o asignaciones para ser desarrolladas en casa.

\section{Conclusiones y recomendaciones}

Se presenta las siguientes conclusiones, resultado de los hallazgos de la investigación: 
1. Los docentes de la Carrera de Letras y Lenguas del CUED de la Sede de Comayagua, que son asumidos como tales, ya sea de planta o por contrato por hora para servir las diferentes asignaturas del Plan de Estudios 1994, manifestaron no haber tenido experiencia laboral en dicha modalidad, siendo el CUED su primer acercamiento a la misma; declararon no haber recibido por parte de la institución, la formación metodológica para desarrollarse en procesos a distancia.

2. El alumno o participante del sistema de educación a distancia se caracteriza, en su mayoría por ser un adulto que de acuerdo con su naturaleza y su circunstancia, tiene compromisos laborales y de familia por lo que encuentra en esta modalidad, la posibilidad de acceder a un título universitario, que no habría podido hacer en formas tradicionales o presenciales. Además se concentra en la sede proveniente de diferentes regiones de la zona central del país. Luego de toda una vida de presencialidad atraviesan dificultades para adaptarse a la responsabilidad que recae sobre él en esta nueva forma de estudio autónomo e independiente.

3. Se constata que la forma de educación a distancia favorece el estudio independiente y autónomo ya que el alumno se acostumbra a estudiar solo y a distancia, es gestor de su propio aprendizaje y aprende a manejar su tiempo y recursos y a estudiar con los recursos que tiene a su alcance.

4. El tutor de Educación a Distancia se encarga de preparar los contenidos, las guías didácticas, los ejercicios y exámenes, y todas las actividades de evaluación de los diferentes espacios pedagógicos, aplicarlas y evaluarlas; así mismo de realizar la función tutorial en una forma mixta: presencial y a distancia.

5. La forma de mediación o de interacción didáctica es mixta, es decir con actividades que se realizan en la visita o tutoría y las que son responsabilidad del estudiante desarrollar en casa. La enseñanza de la Literatura conllevan una variedad de estrategias que van desde aquellas tradicionales como la exposición magistral hasta las más actuales como los proyectos de aula, las investigaciones y talleres y los proyectos de extensión; algunas 
se realizan durante la tutoría o visita, pero la mayor parte del trabajo es realizado en casa bajo la forma de asignaciones.

6. Los textos impresos, las lecturas complementarias y los materiales son todavía imprescindibles en un proceso a distancia y en su mayoría son diseñados por especialistas de la Sección de Letras y Lenguas y son de carácter obligatorio; se utiliza además textos literarios y materiales complementarios pero dichos materiales presentan baja calidad en la reproducción o edición, produciendo quejas de parte de los participantes y muchas dificultades en el logro de los objetivos.

7. El Soporte o sede en donde se realiza la interacción didáctica no cumple con los requerimientos didáctico-pedagógicos para la mediación, produciendo incomodidad, quejas e insatisfacción por parte de los docentes y o alumnos participantes; asimismo, las formas administrativas y de gestión, no satisfacen a los docentes, produciendo quejas e insatisfacción, sobretodo en el docente por contrato por hora.

En cuanto a las recomendaciones, se presenta las siguientes:

1. Con el presente estudio se pretende contribuir con la conceptualización de la educación a distancia, a través de la sistematización del proceso por parte del CUED de la UPNFM a treinta y cuatro años de labor ininterrumpida en la formación de formadores para la educación nacional, con el deseo de que más investigadores emprendan esta labor de documentación de la experiencia y la misma pueda dar pie a estudios complementarios y/o relacionados.

2. Se espera por parte de las autoridades gestoras del sistema, la implementación de estrategias y políticas que lleven al mejoramiento y saneamiento de las condiciones físicas y psicológicas para hacer más cómodo y satisfactorio el escenario en que se desarrolla la interacción didáctica y la relación de docentes-alumnos en el proceso enseñanza-aprendizaje de los estudios literarios, no sólo en la Sede del CUED de Comayagua, sino en todas las sedes en funcionamiento. 
3. Se recomienda la generación de un programa especial de maestría que capacite al personal que labore con la modalidad de educación, en las estrategias de enseñanza en esta modalidad.

4. Será tarea de las coordinaciones de área o especialidad, la formación y actualización constante del personal docente en cuanto a la enseñanza de la lengua y la literatura, acorde con los planes y programas de estudio de las instituciones que incorporarán a los egresados de la UPNFM.

5. Se hace urgente y necesaria la revisión de los materiales y textos utilizados, además de la provisión de mayor, variada y actualizada bibliografía de reconocidas casas editoriales, al servicio de los docentes y estudiantes participantes en los procesos a distancia.

6. Se sugiere la valoración y evaluación constante del desempeño de los encargados de sedes y todo el personal que labora en la gestión y administración de la modalidad de Educación a Distancia. 


\section{Bibliografía}

- [1] Delors, J. (2002). La educación encierra un tesoro. Quito: UNESCO.

- [2] Barberá, Elena; Badia, Antoni; Monimó, Josep M. (2001). La incógnita de la educación a distancia. Barcelona: I.C.E. Universitat Barcelona.

- [3] Noé Martínez, René. (2003). Apuntes sobre educación a distancia. (Texto en proyecto, no publicado).

- [4] Holmberg, Börje. (1981). Educación a distancia: situaciones y perspectivas. Argentina: KAPELUSZ.

- [5] Mena, Marta. (2004). (comp.) La Educación a Distancia en América Latina-Modelos tecnologías y realidades. Buenos Aires: ICDE-UNESCO.

- [6] García Aretio, L. (2007, junio). Un breve apunte histórico. Boletín BENED. (On line), 6. Disponible en http://www.uned.es/ cued/boletin.html

- [7] Arancibia Herrera, M. y H. Pérez San Martín. (2002). Antecedentes Conceptuales, Tecnológicos y Pedagógicos para la Propuesta de un Modelo Educativo a Distancia. Revista de Estudios Pedagógicos (On line), $\mathrm{N}^{\circ} 28$. Disponible en http: / /www.scielo.cl/ scielo.php?scrip=sci_arttext\&pid=S0718. $07052002000100009 \& \operatorname{lng}=e s \& n r m=i s o$

- [8] Sánchez Peralta, Filogonio. (2001). Las tutorías y la construcción de ambientes de aprendizaje en la en la educación abierta y a distancia. Acción Educativa. Revista Electrónica del Centro de Investigaciones y Servicios Educativos. Universidad Autónoma de Sinaloa. Volumen I, Número 1, Febrero del 2001. Culiacán, Sin. México. Disponible en: http://uas.uasnet.mx/ cise/rev/Num1/. 
- [9] Pineda, Alma; Macías Nelly, Turcios, Omar. (2011). El Centro Universitario de Educación a Distancia CUED como respuesta de formación docente en el nivel superior. Tegucigalpa. CUED. (Documento no publicado).

- [10] Carrera de Letras y Lenguas, UPNFM. (2007). Plan de Rediseño de la Carrera de Letras. Documento no publicado.

- [11] Cassany, Daniel; Luna, Martha; Sáenz, Gloria. (1997). Enseñar lengua. Barcelona: GRAÓ.

- [12] Mendoza Fillola, Antonio. (2003). Didáctica de la Lengua y la Literatura. España: Prentice Hall.

- [13] Blanco, Rosa; Astorga, Alfredo; Guadalupe, Cesar; Hevia, Ricardo; Nieto, Margarita; Robalino, Magaly; Rojas, Alfredo. (2007). Educación de calidad para todos: un asunto de derechos humanos. Documento de discusión sobre política educativas en el marco de la II Reunión Intergubernamental del Proyecto Regional de Educación para América Latina y el Caribe (EPT/ $P R E L A C)$. (on line). Disponible en http://portal.unesco.org/geo $\mathrm{g} \quad \mathrm{r}$ a $\mathrm{p} h \mathrm{~h} / \mathrm{e}$ s / e $\mathrm{v}$. $\mathrm{p}$ h $\mathrm{p}$ URL_ID=7910\&URL_DO=DO_TOPIC\&URL_SECTION=201.html

- [14] Viesca Labotón, Maya. (1999, agosto). "Los materiales en la educación a distancia". Revista de Educación y Cultura de la Sección 47 del SNTE, N¹1, UNESCO, Guadalajara, México. (on line). Disponible en http://www.uned.es/catedraunesco-ead/ areas_publicued.htm

- $\quad$ [15] Carpio, Zulia. (2006). Estrategias de aprendizaje (On line). Disponible en http://www.monografias.com/trabajos38/ e s t r a t e g i a s - a p rendiza je / estrategia saprendizaje2.shtml?monosearch

- [16] Reyes Bello, Isadora. (2008). Métodos de recolección de datos (On line). Disponible en: http://www.monografias.com/ trabajos $16 /$ recoleccion-datos/recoleccion datos.shtml 